\title{
Necrolytic Migratory Erythema in a Patient with Neuroendocrine Carcinoma
}

\author{
Michaela Gronau ${ }^{1,2}$, Dirk Jäger ${ }^{1}$, Alexander H. Enk ${ }^{2}$ and Jessica C. Hassel ${ }^{1,2}$
}

\begin{abstract}
Necrolytic migratory erythema is an obligatory paraneoplastic syndrome. Here we describe a patient with a neuroendocrine tumor of probably pancreatic origin. She developed the typical skin lesions under chemotherapy with temozolomide and capecitabine.
\end{abstract}

Key words: mechanoresponsive, necrolytic migratory erythema, neuroendocrine tumor, tumor lysis

(Intern Med 52: 151-152, 2013)

(DOI: 10.2169/internalmedicine.52.8499)

\section{Introduction}

The term necrolytic migratory erythema (NME) was first used by Wilkinson 40 years ago (1). NME is an obligatory paraneoplastic syndrome. Erythematous scaly lesions with centrifugal growth characterize the clinical appearance of the disease. There have been approximately 100 cases of NME published to date, most of which are associated with glucagon-secreting pancreatic tumors. We herein describe the case of a patient with neuroendocrine carcinoma (WHO classification: Ib) of probable pancreatic origin. To our knowledge, three cases of NME associated with neuroendocrine tumors have been previously reported (2-4). We herein describe the first case of NME occurring in association with chemotherapy with temozolomide and capecitabine.

\section{Case Report}

We present the case of a 41-year-old woman with a highly differentiated neuroendocrine carcinoma of probable pancreatic origin first diagnosed three and a half years previously.

The tumor was first misdiagnosed as a malignant SertoliLeydig cell tumor (GII, FIGO IV) in 2007. The diagnosis was revised in June 2010, when the pathologist described the presence of tumor tissue with endocrine structures in the vessels and tumor cells arranged in different sized nests. Immunohistological stainings performed in 2010 showed posi- tivity for pancytokeratin, epithelial membrane antigen, chromogranin, synaptophysin, neuron specific enolase and inhibin-alpha. Calretinin- and CD99-stainings were negative. In addition, a microarray analysis was performed that showed an expression of glucocorticoid receptor and an obvious overexpression of somatostatin receptor 1 (the expression of SSTR 2-5 was negative). The SYP (synaptophysin), CHGA (chromogranin A) and ENO2 (neuron specific enolase) genes showed clear overexpression. A diagnosis of multiple endocrine neoplasia was excluded based on the results of a molecular genetic analysis [no mutations were found in the MEN-1 (MENIN)-gene].

The patient presented with erythematous annular pruritic plaques on the lower legs (Figure) and discrete erythematous scaly plaques on the arms and back. The lesions developed over one week, beginning with a small vesicle with centrifugal growth. Three weeks previously, the patient had received temozolomide and capecitabine to treat the metastasized neuroendocrine tumor with metastases in the liver, diaphragma, vagina and pelvis at last staging.

A biopsy of the skin lesions showed a discrete picture with spongiosis, isolated dyskeratosis, subepidermal edema and a mild perivascular and interstitial lymphocytic infiltrate with sporadic eosinophils. In addition, clusters of cocci bacteria were found, especially at follicular ostia. The serum glucagon level decreased to $31 \mathrm{pg} / \mathrm{mL}$ (ref.: 59-177). Based on the typical clinical picture, medical history and matching histological features, we diagnosed the patient with necrolytic migratory erythema. Oncologic treatment with temo-

${ }^{1}$ National Center for Tumor Diseases Heidelberg, Germany and ${ }^{2}$ Department of Dermatology, Ruprecht Karls-University, Germany Received for publication July 11, 2012; Accepted for publication September 7, 2012

Correspondence to Michaela Gronau, michaela.gronau@med.uni-heidelberg.de 


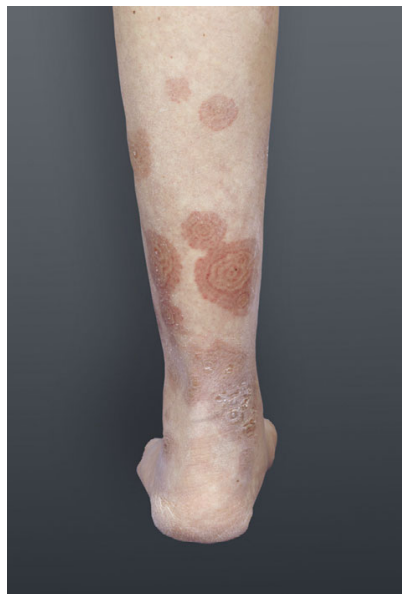

Figure. Clinical Picture

zolomide and capecitabine in combination with local steroids was continued, and the skin lesions disappeared within two weeks. The neuroendocrine tumor was stable under this treatment regimen. The serum chromogranin level decreased from 653 to $48.2 \mathrm{ng} / \mathrm{mL}$ (ref.: <98.1) and the NSE level decreased from 27.3 to $19.1 \mu \mathrm{g} / \mathrm{L}$ (ref.: <17).

The chemotherapy regimen was continued because it was unlikely that the NME was directly caused by treatment with temozolomide and capecitabine. In addition, we continued the treatment because the staging showed that the disease was stable and treating the malignant tumor was given top priority. The skin lesions were asymptomatic and did not significantly bother the patient.

\section{Discussion}

NME is a typical symptom of pancreatic glucagonoma, and there are also cases of NME occurring in association with other types of tumors. The pathogenesis of NME is not clearly understood, and several completely different mechanisms appear to result in the clinical appearance of NME.

A remarkable clinical feature of NME is a symmetric stocking distribution of efflorescences. Mechanical influences and stasis phenomena seem to be cofactors and might explain the typical localization of the disease. Mechanical forces can promote inflammatory responses by activating peripheral terminals of mechanoresponsive afferent sensory neurons (5).

Other conditions known to occur in association with NME, such as liver disease, malabsorptive disorders, ulcerative colitis, Crohn's disease, hepatic cirrhosis, hepatocellular carcinoma, lung cancer and duodenal cancer, should be excluded. Our patient suffered from recurrent hypoglycemia, and the presence of paraneoplastic insulin secretion was supposed. The laboratory data of liver function were within the normal ranges (e.g. GOT: $28 \mathrm{U} / \mathrm{L}$, normal range: $-35 \mathrm{U} / \mathrm{L}$ ). The patient's gastrointestinal function was normal. Although the patient had a low BMI $\left(15.2 \mathrm{~kg} / \mathrm{m}^{2}\right)$ and had lost weight over the previous 10 years $(10 \mathrm{~kg})$, her weight was stable at the time of presentation to our department. There were no signs of zinc deficiency (zinc level: $944 \mu \mathrm{g} / \mathrm{L}$, normal range: $700-1,200 \mu \mathrm{g} / \mathrm{L}$ ) or malnutrition. The total serum protein level was $81.3 \mathrm{~g} / \mathrm{L}$ (normal range: $60-80 \mathrm{~g} / \mathrm{L}$ ) and the albumin level was $51.9 \mathrm{~g} / \mathrm{L}$ (normal range: $30-50 \mathrm{~g} / \mathrm{L}$ ).

Our patient developed NME on day 21 of chemotherapy with temozolomide and capecitabine. Therefore, tumor lysis might have caused an inflammatory response capable of inducing NME. However, due to the pancreatic origin of the neuroendocrine tumor, elevated levels of glucagon cannot be excluded as a cause, even if they were lower in the serum at the time of testing. For patients with NME without evidence of glucagonoma, the expression of pseudoglucagonoma must be established.

In summary, we aimed to emphasize the existence of NME in patients with tumor types other than glucagonoma. These patients often present to dermatologists under a suspicion of chemotherapeutic side effects. Awareness of this rare disease will therefore help to prevent the unnecessary discontinuation of effective oncologic treatments.

The authors state that they have no Conflict of Interest (COI).

\section{References}

1. Wilkinson DS. Necrolytic migratory erythema with pancreatic carcinoma. Proceedings of the Royal Society of Medicine 64: 1179, 1971.

2. Lolis MS, Krishtul A, Vidal C, Shim-Chang H, Phelps R, Lebwohl M. Necrolytic migratory erythema associated with a metastatic neuroendocrine tumor. Cutis 87: 78-80, 2011.

3. Marko PB, Miljkovic J, Zemljic TG. Necrolytic migratory erythema associated with hyperglucagonemia and neuroendocrine hepatic tumors. Acta Dermatovenerol Alp Panonica Adriat 14: 161-164, 166, 2005.

4. Wilkinson SM, Cartwright PH, Allen C, Reeves S, Alexander L, Byrne JP. Necrolytic migratory erythema: association with neuroendocrine tumour with predominant insulin secretion. $\mathrm{Br} \mathrm{J}$ Dermatol 123: 801-805, 1990.

5. Wong VW, Paterno J, Sorkin M, et al. Mechanical force prolongs acute inflammation via T-cell-dependent pathways during scar formation. FASEB J 25: 4498-4510, 2011 Epub 2011/09/12.

(C) 2013 The Japanese Society of Internal Medicine http://www.naika.or.jp/imonline/index.html 